\title{
Ripe or Rotten? Low-Cost Produce Quality Estimation using Reflective Green Light Sensing
}

\section{Zuniga Corrales, Agustin}

2021-07

Zuniga Corrales , A , Flores , H \& Nurmi , P 2021 , ' Ripe or Rotten? Low-Cost Produce Quality Estimation using Reflective Green Light Sensing ', IEEE Pervasive Computing , vol. 20 , no. 3 , pp. 60 - 67 . https://doi.org/10.1109/MPRV.2021.3074474

http://hdl.handle.net/10138/333296

https://doi.org/10.1109/MPRV.2021.3074474

acceptedVersion

Downloaded from Helda, University of Helsinki institutional repository.

This is an electronic reprint of the original article.

This reprint may differ from the original in pagination and typographic detail.

Please cite the original version. 


\title{
Ripe or Rotten? Low-Cost Produce Quality Estimation using Reflective Green Light Sensing
}

\author{
Agustin Zuniga $\dagger$, Huber Flores*, Petteri Nurmi $\dagger$ \\ $\dagger$ Department of Computer Science, University of Helsinki, Helsinki, Finland \\ * Institute of Computer Science, University of Tartu, Estonia
}

\begin{abstract}
We develop an innovative low-cost approach for characterising fresh produce by repurposing inexpensive commercial-off-the-shelf green light sensors for quality estimation. Our approach has been designed to support all stages of the supply chain while at the same time being inexpensive and easy-to-deploy. We validate our approach through extensive empirical benchmarks, showing that it can correctly distinguish organic produce from non-organic items, establish unique fingerprints for different produce, and estimate the quality or ripeness of produce. Specifically, we demonstrate that changes in the reflected green light values correlate with the so-called transpiration coefficients of the produce. We also discuss the practicability of our approach and present application use cases that can benefit from our solution.
\end{abstract}

Index Terms-Pervasive agriculture; Quality Estimation; Wearables; Material Sensing; IoT devices

\section{INTRODUCTION}

Food production and agriculture are increasingly harnessing pervasive computing technologies to improve productivity and to reduce waste throughout the production supply chain. While the main driving force is improved economic profitability, improved efficiency also serves societal development goals through improved food security and reduced food waste. Examples of how pervasive computing can benefit food production and agriculture range from automated monitoring and optimization of growth [1], [2] to automated detection of defects [3], intelligent supply chain management and transport optimization [4], [5], retail inventory management [6], and waste management [7].

Quality estimation is an essential step in food production and agriculture as it helps to mitigate waste and identify problems in the supply chain. Quality estimation is particularly important for low-cost items which have long transportation times but short shelf-life, such as fruits and vegetables. While new mechanisms and strategies to improve the supply chain are constantly being adopted, the short lifespan of items and the complexity of the supply chain make quality assessment highly challenging. Indeed, as illustrated in Figure 1, modern supply chains are highly complex processes that involve a large number of stakeholders. Existing solutions for produce quality assessment, such as (manual) visual inspection [8], automated image processing [9] or different forms of spectral imaging [10], [11], are difficult to adopt as they either support only specific stages of the supply chain or are expensive and difficult to operate. In addition, these techniques mostly provide information about external quality factors, such as

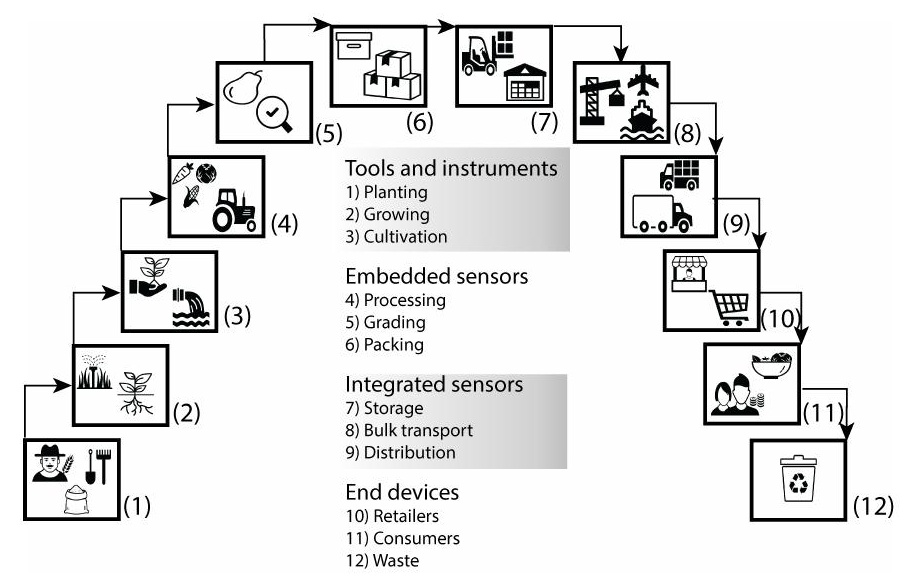

Fig. 1. Use cases for green light sensing in the produce supply chain.

detecting spoilt produce or identifying visual defects, rather than determining the internal quality and remaining shelf-life of the produce. To overcome these limitations, solutions that support the entire supply chain and provide information about internal quality factors are required. To ensure these techniques can be adopted throughout the supply chain, these methods should be inexpensive and easy-to-deploy and operate.

In this article, we present an innovative and low-cost method for characterizing different organic produce using contactbased reflective green light sensing. Our solution repurposes inexpensive and widely available green light sensors, such as those available on smartwatches, to provide insights about internal and external quality factors while only requiring a single point-of-contact - unlike current solutions (see Sec.VI). Reflective green light sensing is an attractive solution for produce quality estimation as the required components are inexpensive, energy-efficient, widely available, and supported by commercial-off-the-shelf devices. These factors make our solution easy to implement and deploy, complementing current techniques. We discuss the general applicability of our approach, and present application use cases that demonstrate its generality across different stages of the supply chain.

We validate our approach through extensive empirical benchmarks that consider a wide range of produce (14 different fruits and vegetables covering all primary classes) and non-organic items. We show that organic produce have clearly distinguishable reflection patterns that can be used to establish an unique fingerprint for each type of produce, and that these fingerprints are highly different from those resulting 
for inorganic produce. Next, we present results from a twoweek long study on product decomposition, demonstrating that changes in reflection patterns correlate with parameters describing internal changes in produce structure and quality. Specifically, we demonstrate that changes in reflectance patterns are related to the transpiration rate coefficient, the transfer coefficient and the respiratory heat generation rate, which are indicators of the pace at which decomposition happens and thus can be used to assess quality of organic products [12]. We also demonstrate the generality of our approach through a follow up study containing a different set of produce from the same 14 product classes as in our primary study. Finally, we demonstrate that reflective green light measurements together with known features of organic produce (scientific name, firmness and epicarp colour) can be used to develop automated quality classification techniques that reach reasonable (up to $83 \%$ in our experiments) accuracy even with the use of simple machine learning techniques.

\section{GReEn Light Sensing In ThE Produce Supply Chain}

We next briefly discuss how our solution can be integrated with the different stages of the supply chain and how it can complement existing practices.

Planting, Growing and Cultivation: In the first stages of the supply chain, we expect green light sensing to be integrated into tools and measurement instruments to provide analytics data to support produce growth, cultivation and harvesting. For example, plots where seeds are planted can use green light sensors to provide analytic data that can be used to determine environmental factors that offer best conditions for produce to grow or the maximum number of produce that can be grown without compromising quality [1]. Our approach can also be integrated with IoT solutions that use humidity, temperature and other sensors to monitor and control growth conditions [2]. Our solution augments these solutions by offering a low-cost approach for monitoring produce quality and firmness. Our approach can also be used to identify diseases that affect the internal consistency of produce as these result in softening of the produce interior - similarly to decomposition.

Processing, Grading, and Packaging: The harvested produce undergo several steps before they are graded, labeled, and packaged for distribution. We envision our solution to be deployed as embedded sensors that are integrated into the machinery responsible for different operations. For example, conveyor belts could integrate green light sensing to detect abnormal produce and supplement vision-based identification of defects [3]. Similarly, our solution can support produce grading by offering insights into the internal quality of the produce instead of relying solely on appearance factors. Our solution can also support the packaging phase by providing input for systems that are responsible for determining pickup and distribution schedules [13].

Storage, Bulk Transport and Distribution: Fresh produce can travel long distances and their distribution is reliant on highly optimized distribution chains. Any disruptions in the distribution chain can significantly degrade product quality, but detecting these changes prior to the product arriving in the destination is challenging. Green light sensing can be embedded inside containers and other packaging to provide information about changes in the produce. This information in turn can be used as input to intelligent supply chain management solutions [5] to optimize air flow and thermal properties inside containers [4], or to identify defects in the refrigeration systems used by the containers. For example, modern supply chains often harvest fruits when they are underripe and control the ripening process by monitoring and reducing ethylene production [14] as this allows extending the storage period and helps to ensure the produce has the correct ripeness when it finally enters the market. Our solution complements these solutions by offering insights into changes in the internal structure of the produce.

Retailers, Consumers, and Waste: Once produce reaches the retailers, it enters the last supply chain phase, where produce is sold, consumed and/or disposed. Retailers can use wearables and sensors integrated into fruit stalls to support product inventory management and to dynamically determine best-before dates [6]. Similarly, consumers can use personal wearables and baskets integrating green light sensors to determine the quality of produce during purchase and consumption phases. Green light sensing can also support disposing operations by helping to separate organic waste from non-organic [7].

\section{Green Light Sensing for Quality Estimation}

The idea in our approach is similar to spectral imaging [10], [11], but we focus solely on green light rather than a wider spectrum of light. The rationale for this is two-fold. Firstly, green light is able to penetrate the surface of most organic products, offering a balance between surface reflection and absorption. Other types of spectral imaging solutions rely either on absorption (e.g., spectroscopy) or reflectance (e.g., hyperspectral imaging) which imposes limitations on their deployment. Green light sensing requires contact with the surface of the object, but can operate using a single point of contact unlike spectroscopes. As part of our experiments, we demonstrate that small gaps between the sensor and produce can be tolerated. Hyperspectral imaging can operate without contact but suffers from a need for a clear view of the objects and high cost of deployment. Secondly, green light sensing units, comprising of sources of green light and a photoreceptor, are affordable and widely available on commercial off-theshelf devices. For example, our experiments rely on a smartwatch that integrates the necessary components (two green light sources and a photoreceptor) and comparable solutions can be easily implemented on low-cost micro controllers.

The processing pipeline of our solution is summarized in Figure 2. The sensing unit is placed in contact of the object that is being measured, and measurements are collected over a short time window (90 seconds in our experiments). The resulting measurements are then filtered, such that we can derive statistical summaries (mean, standard deviation) which provide a fingerprint for the object. The resulting fingerprints can then be used on their own for distinguishing different items - or item classes - or as input for machine learning techniques. 


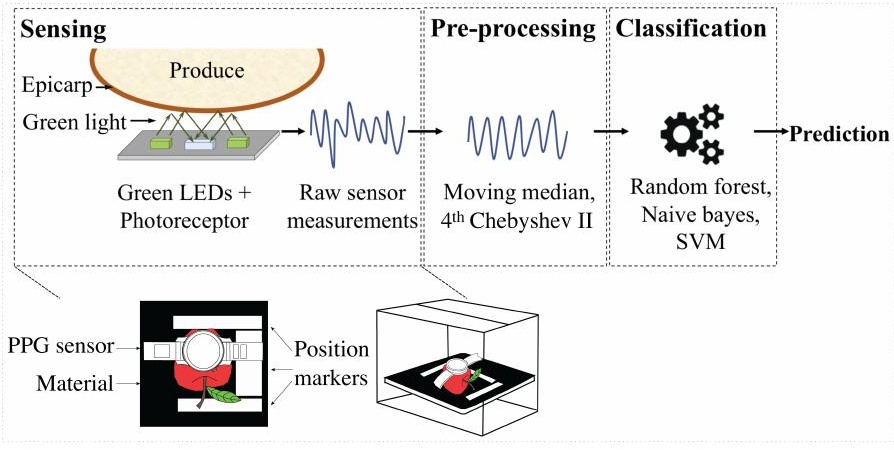

Fig. 2. Processing pipeline for produce quality estimation

\section{EXPERIMENTAL SETUP}

We validate our approach through extensive empirical benchmarks that consider a mixture of inorganic products and fresh produce from all main categories. In the following we describe our experimental setup and measurements in detail.

Objects: We consider fresh produce that are common and cover the main categories of a structural classification of produce: berry (tomato, banana, avocado, kiwi), hesperidium (lemon, mandarine), drupe (mango, peach, plum), pome (pear, apple) and pepo (passion fruit, watermelon, melon). We supplement these with measurements of inorganic objects that are representative of common waste to ensure the measurements are characteristics of the properties of the produce. As examples of inorganic objects, we consider the following manufactured goods: a plastic bottle (polyethylene terephthalate PET), a metal spoon (stainless steel), a small ceramic plate (feldspar), a wooden toy box (solid walnut oak) and a empty bottle of carbonated water (container glass). We use objects that have a clean and appropriated size surface $(>1 \mathrm{~cm} \times 0.5 \mathrm{~cm})$ to reduce possible sampling errors associated with the size of the green light sensor $(0.8 \mathrm{~cm} \times 0.3 \mathrm{~cm})$. For the produce we ensure that they are initially in a fresh and edible state (i.e., no signs of decomposition).

Apparatus: As the green light sensing unit we consider a Samsung Gear S3 Frontier smartwatch. The watch integrates two green LED lights and a photo-receptor, which it uses for measuring heart rate information. We consider the light intensity values reported by the photoreceptor. We rely on a smartwatch instead of a custom hardware prototype as it readily integrates the necessarily components while offering programming support for collecting measurements.

Testbed: We setup a controlled testbed for taking measurements to ensure we can carefully control the positioning of the green light sensors and photoreceptor relative to the materials. Our setup comprises of a black surface with position markers. The fixed markers ensure that all samples are tested approximately in the same area and from the same position, whereas the black surface is used to avoid noise caused by possible background reflections. The green light sensing unit is then placed on top of the sampled object, and a cardboard box is used to cover the testbed to avoid ambient light affecting the measurements. We verified that the luminosity inside the box is zero lux. We separately tested how a change in luminosity (darkness cf. ambient) or distance affects sampling. For each object sample, the sensor takes measurements with $20 \mathrm{~Hz}$ frequency over a 90 second period, where the first and last 15 seconds are removed to avoid possible sources of external light that could have enter into the testbed while setting up the experiment. Note that the 90 second measurement period is solely used to ensure the variance of the measurements is sufficiently small and that our experiments have sufficient statistical power. We have also tested the robustness of the results with different amounts of samples and about 100 samples are necessary to characterize produce with $97.5 \%$ confidence level. These measurements can be taken in about 5 seconds and it is similar for the different materials used in our experiments.

Measurements: We collect measurements following three different experimental designs. First, we collect 5 sets of measurements for each different item (produce and waste) when they are fresh. To mitigate any potential biases resulting from the testbed or placement of objects, each object is first measured once, after which the measurements are repeated using a random item order. Second, we carry out a decomposition experiment where we collect measurements from a single set of produce for a 15 day period and analyze how well variations in light reflectivity capture changes in the surface and characteristics of organic material caused due to decomposition. Finally, we perform a follow-up decomposition experiment where measurements from another set of produce are collected for a 8 day period. The focus of this experiment is to demonstrate the generality of our approach, both in terms of objects to be recognized and the effects of decomposition. In both of the decomposition experiments the produce were kept in room temperature $\left(\approx 21^{\circ} \mathrm{C}\right)$ throughout the experiment.

\section{Results}

\section{A. Characterizing Items}

We first demonstrate that the variations in light intensity across different types of items can be used to establish an unique fingerprint for each item which can be used to distinguish different types of items. We perform this by considering measurement from our first experiment, i.e., 5 sets of measurements for each different type of object (produce or waste) collected during the same day.

The results of the characterization experiment are shown in Figure 3 (left part). Firstly, we can observe clear differences across the objects. We can also observe that manufactured products have lower standard deviation in light reflectivity, which is due to their higher structural consistency. Specifically, the average standard deviation of light for manufactured objects is an order of magnitude smaller than for the organic produce. Skin type and color also had some effect on the results, with items with warmer skin color (yellow, orange, red) having higher surface reflectivity and light intensity values than those with a cool skin color (blue or green).

We used Kruskal-Wallis tests to assess the significance of differences and found the differences to be significant between all the objects $\left(\chi^{2}=62309, \eta^{2}=0.88, \mathrm{p}<0.05\right)$, between manufactured goods and fruits $\left(\chi^{2}=3414, \eta^{2}=0.24\right.$, 


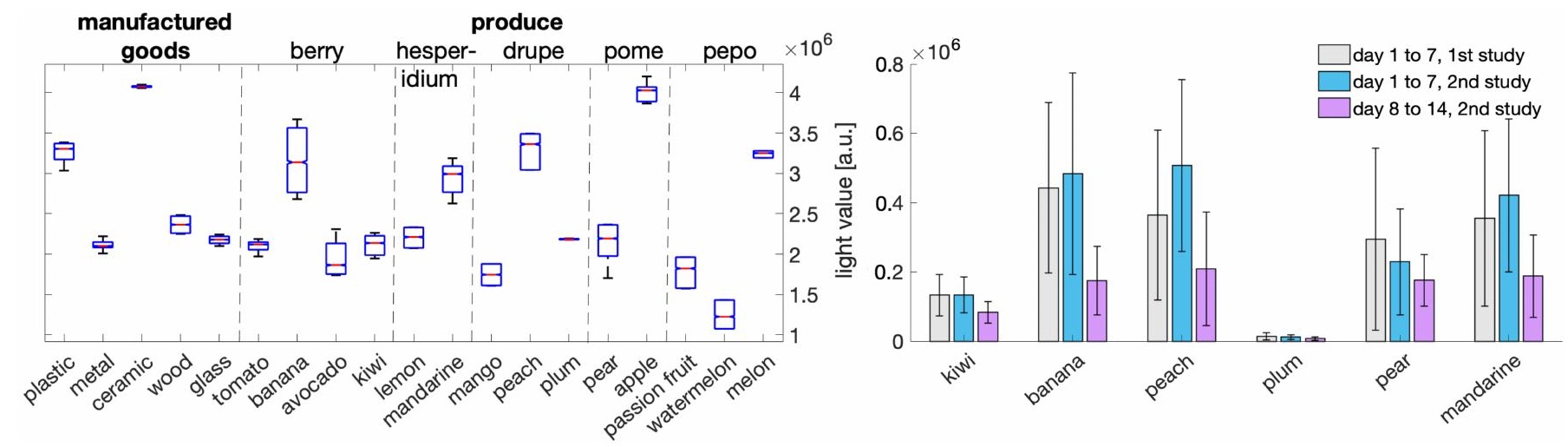

Fig. 3. Characterization of products using green light (left), and the mean absolute deviation of intensity measurements over a 15 days period (right).

$\mathrm{p}<0.05)$, and between different produce categories $\left(\chi^{2}=\right.$ 9744, $\eta^{2}=0.19, \mathrm{p}<0.05$ ). Posthoc comparisons (DunnBonferroni) prove that differences were statistically significant for the vast majority of the objects and produce categories. The sole exceptions were the following pairs: mango-passion fruit $\left(\chi^{2}=-2.2, \mathrm{p}>0.05\right)$, tomato-metal $\left(\chi^{2}=3.2, \mathrm{p}>0.05\right)$, tomato-kiwi $\left(\chi^{2}=-3.4, \mathrm{p}>0.05\right)$ and metal-kiwi $\left(\chi^{2}=0.2\right.$, $\mathrm{p}>0.05)$. The lack of significance between tomato, kiwi and metal is largely due to the sensor being unable to penetrate the surface of the objects. Indeed, all three items are characterized by a low light intensity and small variation, whereas other organic produce exhibit higher variation in the measurements. This issue only occurs when the products are underripe, as ripe and decayed fruits have softer surface which helps the sensor to penetrate the surface of the produce; see Sec. VII

\section{B. Influence of Luminosity and Distance}

We perform a 15 day follow-up experiment using a set of fruits (kiwi, mandarine, plum, pear and passion fruit) that are representative of each produce class ( 2 fruits per class). We collect measurements in two different lighting conditions and at four difference distances: ambient $(32.5 \mathrm{~lx})$, darkness $(0 \mathrm{~lx})$, contact $(0 \mathrm{~cm})$ and three non-contact conditions $(1 \mathrm{~cm}, 5 \mathrm{~cm}$, $10 \mathrm{~cm})$. Friedman test verifies a statistically significant effect of distance on reflectivity values. $\left(\chi^{2}=45, \mathrm{~W}=0.66, \mathrm{p}<\right.$ 0.05). Post-hoc comparisons (Dunn-Bonferroni) indicate that differences are statistically significant after one centimetre. Regarding luminosity, Wilcoxon signed-rank test with different light conditions shows that, when the distance between the sensor and fruit is at most one centimetre, the differences in reflectivity values are not statistically significant $(\mathrm{Z}=-0.24$, $\mathrm{r}=0.02, \mathrm{p}=0.81$ ). In practical terms, this means that the sensor needs to be close to the measured fruit, but that it can accommodate small gaps (up to one centimetre). The result also means that the sensor is insensitive to luminosity when the distance between the sensor and fruit is sufficiently small.

\section{Capturing Quality of Organic Produce}

We next use the measurements from our 15 day decomposition experiment to demonstrate that the fingerprints of different items vary as a function of decomposition. As part of the evaluation, we compare the variations between the first 7 days to the measurements collected from the second decomposition experiment to demonstrate generality of our findings. We also demonstrate that the degree of variations correlates with thermal characteristics of produce. As thermal properties we consider three parameters that are widely used in modeling decomposition risks within different stages of the supply chain: skin mass transfer coefficient $\left(k_{s}\right)$, the transpiration coefficient $\left(k_{t}\right)$ and the respiratory heat generation rate $(W)$ [15]. Skin mass transfer coefficient measures the resistance of the produce to moisture loss [15], transpiration factor measures the loss of water in the produce due to variations in temperature and relative humidity [16] and respiration heat generation rate measures the capacity of the organic material of transforming glucose in water, carbon dioxide and energy [17].

Figure 3 (right) shows the mean absolute deviation of light reflectivity measurements for the two experiments. The magnitude of deviations is strongly linked with changes in the produce during the measurement period and high deviations are observed in fruits that suffer significant variations in the colour and firmness of their skin (e.g., banana, peach). Similarly, produce with more durable skin (e.g., kiwi, plum) witness less significant decay. The speed of decay is dependent on the initial state of the item with, e.g., damage to the skin resulting in faster decomposition patterns. When comparing the results across the two studies, the results are largely similar, with the slight variations likely resulting from variations in the initial state of the produce. We used a Kruskal-Wallis test to verify that differences in measurements between the two decomposition studies were not statistically significant $\left(\chi^{2}=9.93, \eta^{2}=0.35, \mathrm{p}>0.13\right)$.

We also verify that the variations in reflectivity values are representative of organic decay by comparing the parameters describing thermal properties to the difference in mean absolute deviation between two consecutive days. Pearson correlation verifies a significant positive association between the different produce for all three thermal parameters: skin mass transfer coefficient $k_{s}(r=0.96, \mathrm{p}$-value $<0.05$, $B F<0.1)$, transpiration coefficient $k_{t}(r=0.92$, p-value $<0.05, B F<0.1)$ and respiration heat generation rate $W$ $(r=0.92, \mathrm{p}$-value $<0.05, B F<0.1)$. The high correlations suggest that the variations in green light are strongly linked with decomposition of the produce to estimate decay rate. 


\section{Modeling Quality Estimation of Organic Produce}

Lastly, we demonstrate that our approach can support coarse-grained classification of produce into different stages of ripeness. We consider three stages: ripe, overripe and decayed. We evaluate seven different input feature combinations that consider light reflectivity and up to three features characterizing the produce: scientific name, epicarp colour, and firmness. We consider three common and simple classifiers to minimize risks of overfitting and to ensure the models could be easily integrated with low-power devices.

When only light reflectivity is considered, the estimation accuracy is around $60 \%$. This corresponds to a generic model where no information about the type of produce is given. Once we train product-specific models, the performance increases to $70 \%$. The most informative feature is the firmness of the produce, with a model only combining firmness and light intensity resulting in a $74 \%$ average classification accuracy, i.e., models for different types of produce seem to outperform those considering the specific class of produce. As expected, combining multiple features further improves performance with the combination of light reflectivity and all three product characteristics resulting in an average performance of $83 \%$. Of the different classifiers, random forest and SVM have slightly better performance than a naïve Bayes classifier; see Table I] for the averaged confusion matrices over the three classifiers with different feature combinations.

Our experiments aimed at demonstrating the generality and effectiveness of our technique to support different stages of the produce supply chain and, in practice, the way our technique should be adopted depends on the information that is available. For example, if only light intensity values are available, then our approach is best suited for classifying produce or distinguishing organic and inorganic items rather than on providing quality estimates. Indeed, as the results of our first experiment demonstrated, the light intensity fingerprints are unique to produce as long as the light is able to penetrate the skin of the produce. When further information is available, such as a general indication of the firmness of the produce, then coarsegrained quality estimates become feasible - and even better results can be obtained once the name of the produce is also known. In these situations we would expect our solution to be integrated into the supply chain and used to supplement other techniques, e.g., serving as low-cost screening solution for reducing the number of produce that are inspected carefully.

\section{RELATED WORK}

Manual inspection is the most common method for produce quality estimation. While easy to implement, it is highly laborious and inefficient, and vulnerable to human error [8]. The first automated systems for produce quality assessment were based on computer vision, either automatically detecting visual defects or estimating ripeness by examining surface characteristics, such as texture coarseness [3]. These systems are sensitive to illumination and occlusions, requiring unobstructed view of the produce's surface to be able to estimate quality. Manual inspection is predominantly used at producing
TABLE I

AVERAGED CONFUSION MATRICES WITH DIFFERENT FEATURE COMBINATIONS. $L$ : LIGHT REFLECTIVITY VALUE, $N$ : PRODUCE NAME, $E$ : epicarp Colour, $F$ : Firmness, $A$ : ACtual, $P$ : Predicted, $R$ : Ripe, $O$ : OverRipe, $D$ : DECAYED.
$(\mathbf{L})$
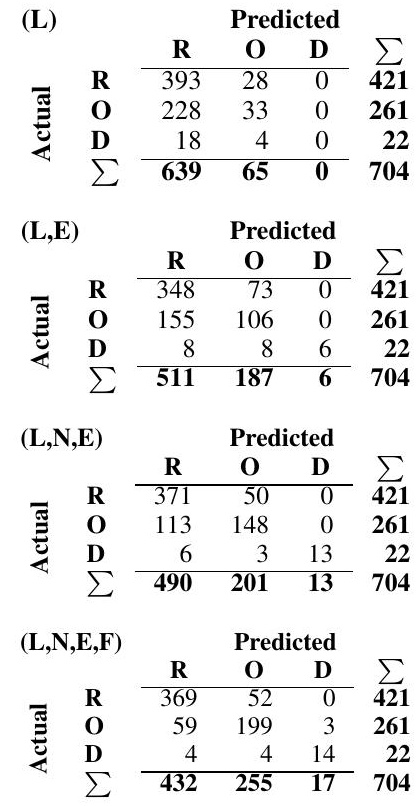
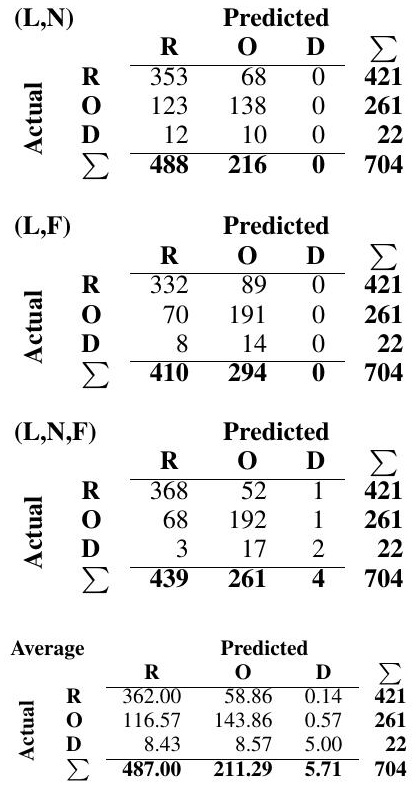

and sales points, whereas computer vision is most useful at the packaging stage. Hence, unlike our method, these techniques cannot be used throughout the supply chain. Unlike our work, these techniques are also limited to external quality factors, such as detecting visual defects, without being able to estimate internal decomposition state and freshness.

Spectral imaging is the main alternative to visual techniques. These techniques range from near infrared (NIR) sensing [10] to hyperspectral imaging [11] - depending on the parts of the light spectrum that are considered. While these approaches capture more details about produce, they require costly and specialized equipment. As these methods effectively extend vision-based techniques by relying on a wider spectrum of light, they inherit the same limitation of being limited to external quality factors. Regulatory food safety inspections rely on spectroscopy which measures absorption of different light wavelengths. Spectroscopic methods are highly accurate and capable of estimating internal quality factors. However, they are highly expensive, require bulky equipment, and taking and analysing measurements is time-consuming, making this approach unsuited for most of the supply chain. Finally, some non-imaging based techniques can be adopted for quality estimation. For example, absorption of wireless signals can be used to estimate water content of produce [18], which in turn correlates with the state of the produce. These techniques are highly sensitive to the measurement setup and can only measure a single item at a time. Our approach overcomes these limitations using a light spectrum that is only partially absorbed, allowing for a measurement setup with a single point-of-contact. Overall, our solution offers a cost-effective alternative that can simultaneously be used to classify different produce and to estimate their quality with the help of off-theshelf green light sensing. 


\section{DISCUSSION}

Aggregate Produce Quality: In the experiments all measurements were collected from a controlled testbed setup and all produce were washed before measuring them. In practice, measurements can be taken from different surface locations and the products can have pieces of soil or other materials on them. The effects of these factors can be mitigated by aggregating measurements from a larger sample size and by considering models with more advanced feature designs. Such data could be collected, e.g., through crowdsourcing or opportunistically from situations where our solution is used together with other techniques.

Food Loss Education: Food loss is a significant environmental concern and reduction of food waste is a leading sustainability goal by the United Nations. Educating people about food loss is critical for reducing food waste and environmental campaigns that address this goal are commonplace in industrialized countries. Our results show that simple green light sensing techniques, integrated into fruit baskets or the personal wearables of people or other modalities can be used to provide hands-on experience of decomposition and thus support educational campaigns.

Limitations and Further Improvements: The results showed that our approach is accurate in characterizing produce as long as the light is capable of penetrating the surface of the object. This mostly concerns fresh produce as any decay results in a softening of the exterior surface of the produce. In these situations, the performance of our technique can be improved using low-cost multispectral imaging, i.e., supplementing the sensor with LEDs operating on different wavelengths. Another option is to rely on a second sensing modality, such as computer vision, to supplement the green light sensing.

\section{SUMmary AND CONCLUSIONS}

We presented an innovative solution that repurposes green light sensors available on commercial off-the-shelf devices for characterizing produce and estimating their quality. Through extensive empirical benchmarks, we demonstrated that different organic produce have unique fingerprints when they are in fresh condition. Through a two-week long study with 18 different items, we demonstrated that the green light fingerprints degrade as products start decomposing, with the magnitude of changes correlation with differences in the produce's transpiration coefficients. We also demonstrated that our results generalize by using a second set of objects to demonstrate that their signatures match with their counterparts in the first set. Finally, we demonstrated that our solution can be used to implement coarse-grained produce quality estimation.

\section{ACKNOWLEDGMENT}

This research is supported by the European Regional Funds through the IT Academy Programme.

\section{REFERENCES}

[1] W.-L. Chen, Y.-B. Lin, Y.-W. Lin, R. Chen, J.-K. Liao, F.-L. Ng, Y.Y. Chan, Y.-C. Liu, C.-C. Wang, C.-H. Chiu et al., "Agritalk: Iot for precision soil farming of turmeric cultivation," IEEE Internet of Things Journal, vol. 6, no. 3, pp. 5209-5223, 2019.

[2] A. Somov, D. Shadrin, I. Fastovets, A. Nikitin, S. Matveev, O. Hrinchuk et al., "Pervasive agriculture: Iot-enabled greenhouse for plant growth control," IEEE Pervasive, vol. 17, no. 4, pp. 65-75, 2018.

[3] A. Bhargava and A. Bansal, "Fruits and vegetables quality evaluation using computer vision: A review," Journal of King Saud UniversityComputer and Information Sciences, 2018.

[4] S. Getahun, A. Ambaw, M. Delele, C. J. Meyer, and U. L. Opara, "Analysis of airflow and heat transfer inside fruit packed refrigerated shipping container: Part i-model development and validation," Journal of food engineering, vol. 203, pp. 58-68, 2017.

[5] A. Pal and K. Kant, "Iot-based sensing and communications infrastructure for the fresh food supply chain," Computer, vol. 51, no. 2, pp. 76-80, 2018.

[6] G. Gaukler, M. Ketzenberg, and V. Salin, "Establishing dynamic expiration dates for perishables: An application of rfid and sensor technology," International Journal of Production Economics, vol. 193, pp. 617-632, 2017.

[7] A. Singh, P. Aggarwal, and R. Arora, "Iot based waste collection system using infrared sensors," in 5th International Conference on Reliability, Infocom Technologies and Optimization. IEEE, 2016, pp. 505-509.

[8] L. Zhou, V. Chalana, and Y. Kim, "Pc-based machine vision system for real-time computer-aided potato inspection," International journal of imaging systems and technology, vol. 9, no. 6, pp. 423-433, 1998.

[9] J. A. Kodagali and S. Balaji, "Computer vision and image analysis based techniques for automatic characterization of fruits-a review," International Journal of Computer Applications, vol. 50, no. 6, 2012.

[10] W. Jiang, G. Marini, N. van Berkel, Z. Sarsenbayeva, Z. Tan, C. Luo, X. He, T. Dingler, J. Goncalves, Y. Kawahara et al., "Probing sucrose contents in everyday drinks using miniaturized near-infrared spectroscopy scanners," Proceedings of the ACM on Interactive, Mobile, Wearable and Ubiquitous Technologies, vol. 3, no. 4, pp. 1-25, 2019.

[11] N.-N. Wang, D.-W. Sun, Y.-C. Yang, H. Pu, and Z. Zhu, "Recent advances in the application of hyperspectral imaging for evaluating fruit quality," Food analytical methods, vol. 9, no. 1, pp. 178-191, 2016.

[12] B. R. Becker, A. Misra, and B. A. Fricke, "Bulk refrigeration of fruits and vegetables part i: theoretical considerations of heat and mass transfer," HVAC\&R Research, vol. 2, no. 2, pp. 122-134, 1996.

[13] M. Bortolini et al., "Fresh food sustainable distribution: cost, delivery time and carbon footprint three-objective optimization," Journal of Food Engineering, vol. 174, pp. 56-67, 2016.

[14] F. Caprioli and L. Quercia, "Ethylene detection methods in post-harvest technology: A review," Sensors and Actuators B: Chemical, vol. 203, pp. 187-196, 2014.

[15] A. Handbook and R. Chapter, "Thermal properties of foods," ASHRAE Refrigeration Handbook, ASHRAE, Atlanta, GA, 2018.

[16] K. Chau, R. Romero, C. Baird, and J. Gaffney, "Transpiration coefficients of fruits and vegetables in refrigerated storage," ASHRAE Report 370-RP, 1987.

[17] S. C. Fonseca, F. A. Oliveira, and J. K. Brecht, "Modelling respiration rate of fresh fruits and vegetables for modified atmosphere packages: a review," Journal of food engineering, vol. 52, no. 2, pp. 99-119, 2002.

[18] H. Huang, L. Liu, and M. O. Ngadi, "Recent developments in hyperspectral imaging for assessment of food quality and safety," Sensors, vol. 14, no. 4, pp. 7248-7276, 2014.

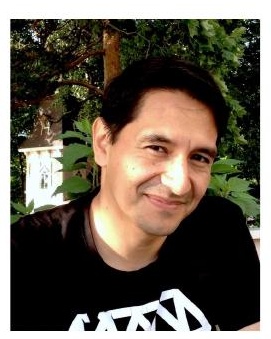

Agustin Zuniga is a doctoral student at the Department of Computer Science, University of Helsinki, Finland. He received his MSc in computer science in 2018 at the University of Helsinki, Finland. His research interests include pervasive computing, Internet of Things, machine learning and data science. Email: agustin.zuniga@helsinki.fi 


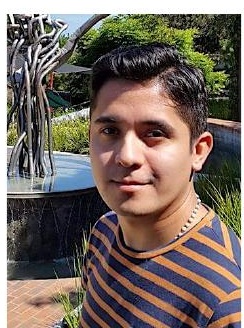

Huber Flores is an Associate Professor with the Institute of Computer Science, University of Tartu, Estonia; and a docent with the Department of Computer Science, University of Helsinki, Finland. He received his $\mathrm{PhD}$ in computer science in 2015 at the University of Tartu, Estonia His research interests include mobile and pervasive computing, distributed systems and mobile cloud computing. Email: huber.flores@ut.ee

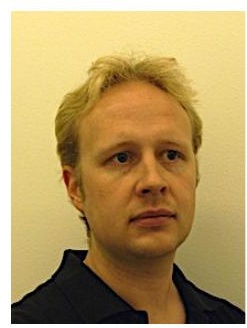

Petteri Nurmi is an Associate Professor at the University of Helsinki and Science, Finland. He received his $\mathrm{PhD}$ in computer science in 2009 at the University of Helsinki, Finland. His research is in the area of pervasive computing and Internet of Things, and is characterized by a focus on research questions surrounding the collection, analysis and use of sensor data. Email: petteri.nurmi@helsinki.fi 